\title{
LIVŠIC THEOREM FOR LOW-DIMENSIONAL DIFFEOMORPHISM COCYCLES
}

\author{
ALEJANDRO KOCSARD AND RAFAEL POTRIE
}

\begin{abstract}
We prove a Livšic type theorem for cocycles taking values in groups of diffeomorphisms of low-dimensional manifolds. The results hold without any localization assumption and in very low regularity. We also obtain a general result (in any dimension) which gives necessary and sufficient conditions to be a coboundary.
\end{abstract}

\section{INTRODUCTION}

In the study of hyperbolic dynamical systems there is a general (and vague) idea that can be summarized with the following sentence:

Most of the dynamical interesting information on a hyperbolic system is concentrated in its periodic orbits.

An archetypal example of a result supporting this idea is the celebrated Livšic's theorem [Liv71, Liv72] claiming that given a hyperbolic homeomorphism $f: M ๖$, a Hölder function $\Phi: M \rightarrow \mathbb{R}$ is a coboundary, i.e. there exists a continuous function $u: M \rightarrow \mathbb{R}$ satisfying

if and only if

$$
u \circ f-u=\Phi,
$$

$$
\sum_{j=0}^{n-1} \Phi\left(f^{j}(p)\right)=0,
$$

for every periodic point $p \in M$, with $f^{n}(p)=p$.

Due to the interest this result has received since its appearance and the large amount of consequences that follow from it, several generalizations have been studied. Some of them consider more general dynamics on the base. For instance, in the works [KK96, Wil13] the cohomology of real cocycles over partially hyperbolic systems is analyzed.

In this paper, we consider a different kind of generalization: given a complete metric group $G$, a $G$-cocycle is just a continuous map $\Phi: M \rightarrow G$ and one wants to determine whether the condition

$$
\Phi\left(f^{n-1}(p)\right) \Phi\left(f^{n-2}(p)\right) \ldots \Phi(p)=e_{G}, \quad \forall p \in \operatorname{Fix}\left(f^{n}\right),
$$

where $e_{G}$ is the identity element of $G$, is not just necessary but also sufficient to guaranty the existence of a "transfer function" $u: M \rightarrow G$ satisfying

$$
\Phi(x)=u(f(x)) u(x)^{-1}, \quad \forall x \in M .
$$

Livšic himself gave in [Liv71] an affirmative answer to this question for cocycles taking values on a topological group admitting a complete bi-invariant distance (e.g. Abelian or compact groups). However, the general situation is considerably much more complicated.

So far, the main technique to handle this problem when the group $G$ does not admit a bi-invariant metric has consisted in considering a left-invariant metric on

Date: March 14, 2018. 
$G$ and to try to control the distortion produced by right translations to be able to apply the very same scheme of proof used in the Abelian case.

In order to get such a control of the distortion of the distance, some localization hypotheses have been considered in the literature. For instance, in [Liv72] Livšic gave a positive answer to above question for linear cocycles (i.e. where $G=\mathrm{GL}_{d}(\mathbb{R})$ ) which are not too far away from the identity constant cocycle. Improvements of these results using weaker localization hypotheses have been obtained for cocycles taking values in arbitrary finite-dimensional Lie groups (see [dlLW10, KN11] and references therein) until the recent complete solution of the global Livšic problem for linear cocycles [Kal11] (see also the recent preprint [GG14]).

In the infinite dimensional case, particularly when $G$ is a group of diffeomorphisms of a compact manifold, the study began with the seminal paper of Niţică and Török [NT95]. The diffeomorphism groups seem to be the most interesting infinite dimensional groups for applications to rigidity theory (see [KN11] and references therein).

It is important to remark that, in contrast with the finite dimensional case, all the results for groups of diffeomorphisms obtained so far (see [dlLW10] for a survey with references) involve non-sharp localization hypotheses (in the sense that not every coboundary satisfies them) and require higher regularity for the diffeomorphism group (i.e. $G=\operatorname{Diff}^{r}(N)$, with $r \geqslant 4$ ). Moreover, the control of distortion techniques used in [NT95, dlLW10] yield a loss of regularity in the solution of the cohomological equation. A recent result for infinite dimensional groups which does not fit in the previous description is due to Navas and Ponce [NP13]. They prove a Livšic theorem for cocycles taking values in the group of analytic germs at the origin.

In this paper we use completely different techniques, with a more geometric flavor, which allow us to deal with the low regularity case (cocycles can take values in the group of $C^{1}$-diffeomorphisms). The main novelty of our approach is Theorem 3.1 which can be of independent interest. Regarding this result within the context of localization arguments, we could say that it is proven that our non-uniform localization hypothesis (i.e. vanishing of fibered Lyapunov exponents) is equivalent to be a coboundary.

In $\S 4$ we show that in the low-dimensional case the periodic orbit condition implies the vanishing of fibered Lyapunov exponents, proving in such cases the general (or global) Livšic theorem for groups of diffeomorphisms. We conjecture that such a result holds in any dimension.

1.1. Main results. The main results of this article are the following Livšic type theorems which, to the best of our knowledge, are the first general (i.e. global) results for cocycles taking values in groups of diffeomorphisms of compact manifolds:

Theorem A. Let $f: M \bigcirc$ be a hyperbolic homeomorphism and $\Phi: M \rightarrow \operatorname{Diff}^{1}(\mathbb{R} / \mathbb{Z})$ be an $\alpha$-Hölder cocycle satisfying the so called periodic orbit obstructions

$$
\Phi\left(f^{n-1}(p)\right) \circ \Phi\left(f^{n-2}(p)\right) \circ \cdots \circ \Phi(p)=i d_{\mathbb{R} / \mathbb{Z}}, \quad \forall p \in \operatorname{Fix}\left(f^{n}\right), \quad \forall n \in \mathbb{N} .
$$

Then, there exists an $\alpha$-Hölder map $u: M \rightarrow \operatorname{Diff}^{1}(\mathbb{R} / \mathbb{Z})$ satisfying

$$
\Phi(x)=u(f(x)) \circ u(x)^{-1}, \quad \forall x \in M .
$$

Remark 1.1. The very same argument we use to prove Theorem A works for cocycles taking values in $\operatorname{Diff}^{1}([0,1])$

For higher regularity, invoking the main result of [dlLW11], one easily gets the following consequence of Theorem A: 
Corollary 1.2. Let $f: M \bigcirc$ be a hyperbolic homeomorphism and $\Phi: M \rightarrow \operatorname{Diff}^{r}(\mathbb{R} / \mathbb{Z})$, with $r \geqslant 1$, be an $\alpha$-Hölder cocycle satisfying the periodic orbit obstructions.

Then, there exists an $\alpha$-Hölder map $u: M \rightarrow \operatorname{Diff}^{r}(\mathbb{R} / \mathbb{Z})$ satisfying

$$
\Phi(x)=u(f(x)) \circ u(x)^{-1}, \quad \forall x \in M .
$$

Assuming higher regularity on the dynamics of the base and the cocycle, applying the results of [Jou88, NT96] one can improve the regularity of the solution of the cohomological equation. Since this kind of results is beyond the scope of this article, we suggest the interested reader to consult [KN11, Wil13] for further information.

In dimension 2, we can obtain a similar result for the group of area-preserving diffeomorphisms:

Theorem B. Let $M$ be a smooth closed manifold and $f: M \bigcirc$ be a $C^{1+\theta}$ transitive Anosov diffeomorphism. Let $S$ denote a compact surface, $\omega$ be a Lebesgue probability measure on $S$ and let $\operatorname{Diff}_{\omega}^{r}(S)$ be the group of $C^{r}$-diffeomorphisms of $S$ that leave $\omega$ invariant.

Let $\Phi: M \rightarrow \operatorname{Diff}_{\omega}^{1+\alpha}(S)$ be a $C^{1+\alpha}-$ cocycle ${ }^{1}$ satisfying the periodic orbit obstructions.

Then, there exists an $\alpha$-Hölder map $u: M \rightarrow \operatorname{Diff}_{\omega}^{1}(S)$ such that

$$
\Phi(x)=u(f(x)) \circ u(x)^{-1}, \quad \forall x \in M .
$$

The proofs of Theorems A and B consist in two steps. The first one concerns the vanishing of Lyapunov exponents for the cocycles and relies heavily in the lowdimensionality of the fibers. The second one holds in any dimension and can be of independent interest (see Theorem 3.1).

Acknowledgments. We are grateful to A. Navas and M. Ponce for useful discussions and bringing these problems to our attention. This paper was finished during a visit of A.K. to Universidad de la República (Uruguay).

A.K. is very thankful to R. Markarian for his hospitality. He was partially supported by CNPq and FAPERJ (Brasil), and Fondo Clemente Estable (Uruguay).

R.P. was partially supported by CSIC group 618, FCE-3-2011-1-6749 and Balzan's research project of J.Palis.

\section{Preliminaries AND notations}

2.1. Hölder continuity. All along this paper, $(M, d)$ will denote a compact metric space. If $\left(M^{\prime}, d^{\prime}\right)$ denotes another arbitrary metric space and $0<\alpha \leqslant 1$, a map $\psi: M \rightarrow M^{\prime}$ is said to be $\alpha$-Hölder whenever

$$
|\psi|_{\alpha}:=\sup _{x \neq y} \frac{d^{\prime}(\psi(x), \psi(y))}{d(x, y)^{\alpha}}<\infty .
$$

Most of the functions and maps we shall deal with in this paper will be at least Hölder because, as it was already observed in [Koc13], in general $C^{0}$ regularity is not appropriate for dynamical cohomology.

When $\alpha<1$, the space of $\alpha$-Hölder maps from $M$ to $M^{\prime}$ will be denoted by $C^{\alpha}\left(M, M^{\prime}\right)$. As usual, we use the term Lipschitz as a synonym of 1-Hölder, and to avoid confusions with the differentiable case, we shall write $C^{\operatorname{Lip}}\left(M, M^{\prime}\right)$ for the space of Lipschitz functions.

For such a real constant $\alpha$, we can define a new distance on $M$ by

$$
d_{\alpha}(x, y):=d(x, y)^{\alpha}, \quad \forall x, y \in M .
$$

Observe that the topologies induced by $d$ and $d_{\alpha}$ coincide and a map $\psi:(M, d) \rightarrow$ $\left(M^{\prime}, d^{\prime}\right)$ is $\alpha$-Hölder if and only if $\psi:\left(M, d_{\alpha}\right) \rightarrow\left(M^{\prime}, d^{\prime}\right)$ is Lipschitz.

\footnotetext{
${ }^{1}$ This means that the induced map $M \times S \ni(x, y) \mapsto \Phi(x)(y) \in S$ is $C^{1+\alpha}$.
} 
2.2. Borel probability measures. Given an arbitrary locally compact metric space $X$, we write $\mathfrak{M}(X)$ for the space of Borel probability measures on $X$ and we will always consider it endowed with (the restriction of) the weak- $\star$ topology. If $Y$ denotes another compact metric space, any continuous map $h: X \rightarrow Y$ naturally induces a linear map $h_{\star}: \mathfrak{M}(X) \rightarrow \mathfrak{M}(Y)$ defined by the following property:

$$
\int_{Y} \phi \mathrm{d}\left(h_{\star} \mu\right):=\int_{X} \phi \circ h \mathrm{~d} \mu, \quad \forall \phi \in C_{c}^{0}(Y), \forall \mu \in \mathfrak{M}(X) .
$$

In this way, if $f: X \supset$ is a continuous map, one defines the space of $f$-invariant measures by

$$
\mathfrak{M}(f):=\left\{\mu \in \mathfrak{M}(M): f_{\star} \mu=\mu\right\} .
$$

2.3. Hyperbolic homeomorphisms. Let $(M, d)$ be a compact metric space and $f: M ๖$ be a homeomorphism. Given any $x \in M$ and $\epsilon>0$, define the local stable and unstable sets by

$$
\begin{aligned}
& W_{\varepsilon}^{s}(x, f):=\left\{y \in M: d\left(f^{n}(x), f^{n}(y)\right) \leqslant \varepsilon, \forall n \geqslant 0\right\}, \\
& W_{\varepsilon}^{u}(x, f):=\left\{y \in M: d\left(f^{n}(x), f^{n}(y)\right) \leqslant \varepsilon, \forall n \leqslant 0\right\},
\end{aligned}
$$

respectively. Where there is no risk of ambiguity, we just write $W_{\epsilon}^{s}(x)$ instead of $W_{\epsilon}^{s}(x, f)$, and the same holds for the local unstable set.

Following [AV10], we introduce the following

Definition 2.1. A homeomorphism $f: M \oslash$ is said to be hyperbolic with local product structure whenever there exist constants $\varepsilon_{0}, \delta_{0}, K_{0}, \lambda>0$ and functions $\nu_{s}, \nu_{u}: M \rightarrow(0, \infty)$ such that the following conditions are satisfied:

(h1) $d\left(f\left(y_{1}\right), f\left(y_{2}\right)\right) \leqslant \nu_{s}(x) d\left(y_{1}, y_{2}\right), \forall x \in M, \forall y_{1}, y_{2} \in W_{\varepsilon_{0}}^{s}(x)$;

(h2) $d\left(f\left(y_{1}\right), f\left(y_{2}\right)\right) \geqslant \nu_{u}(x) d\left(y_{1}, y_{2}\right), \forall x \in M, \forall y_{1}, y_{2} \in W_{\varepsilon_{0}}^{u}(x)$;

(h3) $\nu_{s}^{(n)}(x):=\nu_{s}\left(f^{n-1}(x)\right) \ldots \nu_{s}(x)<K_{0} e^{-\lambda n}, \forall x \in M, \forall n \geqslant 1$;

(h4) $\nu_{u}^{(n)}(x):=\nu_{u}\left(f^{n-1}(x)\right) \ldots \nu_{u}(x)>K_{0} e^{\lambda n}, \forall x \in M, \forall n \geqslant 1$;

(h5) If $d(x, y) \leqslant \delta_{0}$, then $W_{\varepsilon_{0}}^{u}(x)$ and $W_{\varepsilon_{0}}^{s}(y)$ intersect in a unique point which is denoted by $[x, y]$, and it depends continuously on $x$ and $y$.

Remark 2.2. For the sake of simplicity of the exposition and to avoid unnecessary repetitions, from now on we shall assume that all hyperbolic homeomorphisms are transitive and exhibit local product structure.

For such homeomorphisms, one can define the stable and unstable sets by

$$
W^{s}(x, f):=\bigcup_{n \geqslant 0} f^{-n}\left(W_{\epsilon}^{s}\left(f^{n}(x)\right)\right) \text { and } W^{u}(x, f):=\bigcup_{n \geqslant 0} f^{n}\left(W_{\epsilon}^{u}\left(f^{-n}(x)\right)\right),
$$

respectively.

Notice that shifts of finite type and basic pieces of Axiom A diffeomorphisms are particular examples of hyperbolic homeomorphisms with local product structure (see for instance [Mañ87, Chapter IV,§9] for details).

Remark 2.3. For our purposes, it is important to notice that the notion of hyperbolicity for homeomorphisms is invariant under Hölder changes of metric. More precisely, a homeomorphism $f:(M, d) \supset$ is hyperbolic if and only $f:\left(M, d_{\alpha}\right) \supset$ is hyperbolic, for any $\alpha \in(0,1)$, where the distance $d_{\alpha}$ is defined by (1).

The following result is proven for locally maximal hyperbolic sets of smooth diffeomorphisms in [KH96, Chapter 6]. However, by inspection on the proof (see [KH96, Corollary 6.4.17 and Proposition 6.4.16 ]) it can be easily check that the very same proof works for hyperbolic homeomorphisms with local product structure (see also [Kal11, page 1026]): 
Theorem 2.4 (Anosov closing lemma). Let $f:(M, d) \bigcirc$ be a hyperbolic homeomorphism ${ }^{2}$. Then, there exist constants $c, \delta_{1}>0$ such that for every $x \in M$ and any $n>0$ satisfying $d\left(x, f^{n}(x)\right)<\delta_{1}$, there exist unique points $p \in \operatorname{Fix}\left(f^{n}\right)$ and $y \in M$ such that:

(1) $d\left(f^{i}(x), f^{i}(p)\right) \leqslant c d\left(x, f^{n}(x)\right) e^{-\lambda \min \{i, n-i\}}$;

(2) $d\left(f^{i}(p), f^{i}(y)\right) \leqslant c d\left(x, f^{n}(x)\right) e^{-\lambda i}$;

(3) $d\left(f^{i}(x), f^{i}(y)\right) \leqslant c d\left(x, f^{n}(x)\right) e^{-\lambda(n-i)}$;

for every $i \in\{0, \ldots, n-1\}$, where $\lambda>0$ is the constant given in Definition 2.1.

Remark 2.5. Notice that by uniqueness, we have that $y=[x, p]$, where the brackets $[\cdot, \cdot]$ are given by Definition 2.1.

2.4. Cocycles and coboundaries. Let $G$ denote a topological group whose topology is induced by a complete distance function $d_{G}$, and let $f:(M, d) Ð$ be a homeomorphism.

In this work all cocycles we consider will be at least continuous. In fact, a $G$ cocycle (over $f$ ) is just a continuous map $\Phi: M \rightarrow G$. As usual, we use the following notation

$$
\Phi^{(n)}(x):= \begin{cases}e_{G}, & \text { if } n=0 \\ \Phi\left(f^{n-1}(x)\right) \Phi^{(n-1)}(x), & \text { if } n>0 \\ \left(\Phi^{(-n)}\left(f^{n}(x)\right)\right)^{-1}, & \text { if } n<0\end{cases}
$$

where $e_{G} \in G$ denotes the identity element of $G$. We say that $\Phi$ is a Hölder cocycle when $\Phi:(M, d) \rightarrow\left(G, d_{G}\right)$ is an $\alpha$-Hölder map, for some $\alpha \in(0,1]$.

A $G$-cocycle $\Phi$ is said to be a $G$-coboundary when there exists a continuous map $u: M \rightarrow G$ such that

$$
\Phi(x)=u(f(x)) \cdot(u(x))^{-1}, \quad \forall x \in M .
$$

Notice that in this case it clearly holds $\Phi^{(n)}(x)=u\left(f^{n}(x)\right) \cdot(u(x))^{-1}$, for any $n \in \mathbb{Z}$ and any $x \in M$.

The first natural family of obstructions one encounters for a $G$-cocycle to be a $G$-coboundary is called periodic orbit obstructions (just POO for short):

$$
\Phi^{(n)}(p)=e_{G}, \quad \forall n \geqslant 1, \forall p \in \operatorname{Fix}\left(f^{n}\right) .
$$

In this work we shall mainly concentrate in the case where $G=\operatorname{Diff}^{1}(N)$. To deal with this case, we need a slight generalization of the concept of cocycles that we introduce in the following paragraph.

2.5. Fiber bundle maps and cocycles. Let $N$ denote a compact differentiable manifold and $(M, d)$ be compact metric space as above. Given any $\alpha \in(0,1]$ and $r \geqslant 0$, a $C^{\alpha, r}$-fiber bundle over $M$ with fiber $N$ is an object $N \rightarrow \mathcal{E} \stackrel{\pi}{\rightarrow} M$, where $\mathcal{E}$ is a topological space and $\pi$ is a surjective $\alpha$-Hölder map such that there exists a finite open cover $\left\{U_{j}\right\}_{1}^{n}$ of $M$ with the following properties:

- For each $j \in\{1, \ldots, n\}$, there exists a homeomorphism $\varphi_{j}: \pi^{-1}\left(U_{j}\right) \rightarrow$ $U_{j} \times N$

- If $U_{i} \cap U_{j} \neq \varnothing$, there is an $\alpha$-Hölder map $g_{i j}: U_{i} \cap U_{j} \rightarrow \operatorname{Diff}^{r}(N)$ such that

$$
\varphi_{i} \circ \varphi_{j}^{-1}(x, y)=\left(x,\left(g_{i j}(x)\right)(y)\right), \quad \forall(x, y) \in\left(U_{i} \cap U_{j}\right) \times N .
$$

As usual, one defines the fiber over $x$ by $\mathcal{E}_{x}:=\pi^{-1}(x) \subset \mathcal{E}$ and, due to the fiber bundle properties, it can naturally endowed with a $C^{r}$-differentiable structure turning it into a $C^{r}$-manifold $C^{r}$-diffeomorphic to $N$.

\footnotetext{
${ }^{2}$ Remember that by our assumption, hyperbolic homeomorphisms have local product structure.
} 
As usual, when $N=\mathbb{R}^{d}$ and the change of coordinate maps $g_{i j}$ given above are $\alpha$-Hölder and have their image contained in $\mathrm{GL}_{d}(\mathbb{R})$, we say that $\mathbb{R}^{d} \rightarrow \mathcal{E} \stackrel{\pi}{\rightarrow} M$ is a $C^{\alpha}$-vector bundle.

The total space of any $C^{\alpha, r}$-fiber bundle $N \rightarrow \mathcal{E} \stackrel{\pi}{\rightarrow} M$ can be endowed with a distance function $d_{\mathcal{E}}$ constructed as follows:

Let $d_{N}$ be a distance function compatible with the smooth structure of the fiber manifold $N,\left\{U_{j}\right\}_{1}^{n}$ and $\left\{\varphi_{j}\right\}_{1}^{n}$ be a local trivialization atlas as above, $L>0$ be the Lebesgue number of the open covering $\left\{U_{j}\right\}_{1}^{n}$ and define

$$
d_{\mathcal{E}}(\zeta, \eta):=\min \left\{d(\pi(\zeta), \pi(\eta))+\inf _{1 \leqslant j \leqslant n}\left\{d_{N}\left(\operatorname{pr}_{2}\left(\varphi_{j}(\zeta)\right), \operatorname{pr}_{2}\left(\varphi_{j}(\eta)\right)\right)\right\} ; L\right\}
$$

where $\operatorname{pr}_{2}: M \times N \rightarrow N$ denotes the projection on the second coordinate, and by convention, we declare that $d_{N}\left(\operatorname{pr}_{2}\left(\varphi_{j}(\zeta)\right), \operatorname{pr}_{2}\left(\varphi_{j}(\eta)\right)\right)=\operatorname{diam}_{d_{N}} N$ whenever $\zeta$ or $\eta$ does not belong to $\pi^{-1}\left(U_{j}\right)$.

A Riemannian structure on a $C^{\alpha, r}$-fiber bundle $\mathcal{E}$ consists on choosing a Riemannian metric on each fiber $\mathcal{E}_{x}$ which varies Hölder-continuously with $x \in M$.

From now on, we will assume every fiber bundle is endowed with a fixed Riemannian structure and a distance function constructed as above. It is important to remark that all the concepts we will consider about fiber bundles are completely independent of these chosen structures.

In this setting, given another $C^{\alpha, r}$-fiber bundle $N \rightarrow \tilde{\mathcal{E}} \stackrel{\tilde{\pi}}{\rightarrow} M$ and a $C^{\alpha}$ homeomorphism $f: M \circlearrowleft$, a $C^{\alpha, r}$-bundle map over $f$ is a homeomorphism $F: \mathcal{E} \rightarrow$ $\tilde{\mathcal{E}}$ satisfying $\tilde{\pi} \circ F=f \circ \pi$ and such that the map $F_{x}:=\left.F\right|_{\mathcal{E}_{x}}: \tilde{\mathcal{E}}_{x} \rightarrow \mathcal{E}_{f(x)}$ is a $C^{r}$-diffeomorphism, for every $x \in M$.

As usual, the fiber bundle $N \rightarrow \mathcal{E}=M \times N \stackrel{\mathrm{pr}_{1}}{\longrightarrow} M$, where $\mathrm{pr}_{1}: M \times N \rightarrow M$ denotes the projection on the first coordinate, is said to be trivial.

In this case, any $C^{\alpha} \operatorname{Diff}^{r}(N)$-cocycle $\Phi: M \rightarrow \operatorname{Diff}^{r}(N)$ naturally induces a $C^{\alpha, r}$-bundle map over any $f \in \operatorname{Homeo}(M)$ defining $F=F_{\Phi, f}: M \times N \oslash$ by

$$
F(x, y):=(f(x), \Phi(x)(y)), \quad \forall(x, y) \in M \times N
$$

Observe that in such a case $F_{x}=\Phi(x)$, for every $x \in M$.

Such a particular bundle map is usually called the skew-product induced by $\Phi$ and $f$. So, bundle maps can be considered as generalizations of cocycles taking values in groups of diffeomorphisms.

2.6. POO and coboundaries for bundle maps. We can easily extends the notion of periodic orbit obstructions to fiber bundle maps on fiber bundles which, a priori, are not necessarily trivial:

A bundle map $F: \mathcal{E} \oslash$ over $f: M ๖$ is said to satisfy the periodic orbit obstructions whenever, for every $n \geqslant 1$ it holds

$$
F_{p}^{n}(\zeta)=\zeta, \quad \forall p \in \operatorname{Fix}\left(f^{n}\right), \forall \zeta \in \mathcal{E}_{p}
$$

Now we finish this paragraph extending the notion of coboundary for ( $\grave{a}$ priori more general) fiber bundle maps: if $N \rightarrow \mathcal{E} \stackrel{\pi}{\rightarrow} M$ denotes a $C^{\alpha, r}$-fiber bundle, a $C^{\alpha, r}$-bundle map $F: \mathcal{E} \bigcirc$ is said to be a $C^{\alpha, s}$-coboundary, with $s \leqslant r$, when there exists a $C^{\alpha, s}$-bundle map $H: \mathcal{E} \rightarrow M \times N$ over the identity map $i d: M \circlearrowleft$ such that the following diagram commutes: 


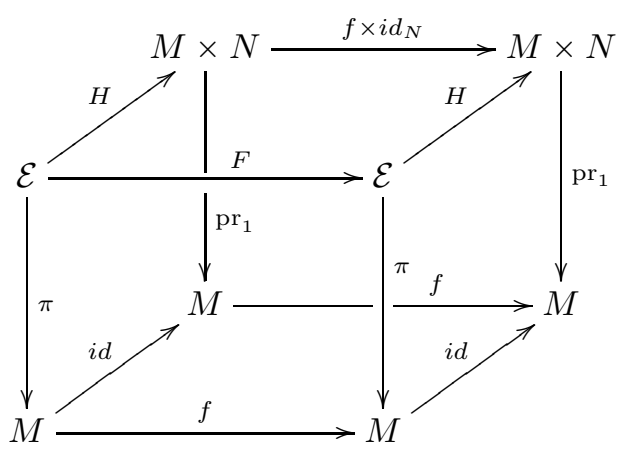

Observe that with our definition, any fiber bundle admitting a coboundary bundle map is à posteriori trivial (see Theorem 3.1 for details and a motivation for this definition).

2.7. Lyapunov exponents for bundle maps. Let $\pi: \mathcal{E} \rightarrow M$ be a $C^{\alpha, r}$-fiber bundle (endowed with a Riemannian structure) and consider a $C^{\alpha, r}$-bundle map $F: \mathcal{E} \circlearrowright$ over $f: M \circlearrowright$. Given any point $\zeta \in \mathcal{E}$ and $n \in \mathbb{Z}$, we have the linear map

$$
D\left(\left.F^{n}\right|_{\mathcal{E}_{\pi(\zeta)}}\right)_{\zeta}: T_{\zeta} \mathcal{E}_{\pi(\zeta)} \rightarrow T_{F^{n}(\zeta)} \mathcal{E}_{f^{n}(\pi(\zeta))}
$$

between normed vector spaces, and hence it makes sense to talk about its norm. For the sake of simplicity, such linear operator will be just denoted by $\partial_{\mathrm{fib}} F^{n}(\zeta)$. by

Then, one defines the extremal Lyapunov exponents of $F$ along the fibers at $\zeta \in \mathcal{E}$

$$
\begin{aligned}
& \lambda^{+}(F, \zeta)=\lim _{n \rightarrow+\infty} \frac{1}{n} \log \left\|\partial_{\mathrm{fib}} F^{n}(\zeta)\right\| ; \\
& \lambda^{-}(F, \zeta)=\lim _{n \rightarrow+\infty} \frac{1}{n} \log \left\|\left(\partial_{\mathrm{fib}} F^{n}(\zeta)\right)^{-1}\right\|^{-1} ;
\end{aligned}
$$

whenever these limits exist. As a consequence of the sub-additive ergodic theorem, it is well-known that theses limits exist almost-everywhere with respect to any $F$-invariant probability measure.

Given any $\hat{\mu} \in \mathfrak{M}(F)$, one defines the extremal Lyapunov exponents of $F$ with respect to $\hat{\mu}$ by

$$
\lambda^{ \pm}(F, \hat{\mu}):=\int_{\mathcal{E}} \lambda^{ \pm}(F, \zeta) \mathrm{d} \hat{\mu}(\zeta) .
$$

The topological version of the invariance principle of Avila and Viana [AV10] gives strong consequences of vanishing of the extremal Lyapunov exponents of certain invariant measures.

2.8. Dominated bundle maps. As above, let us suppose $F: \mathcal{E} \supset$ is a $C^{\alpha, r_{-}}$ bundle map over a transitive hyperbolic homeomorphism $f: M ๖$. Given $\beta>0$, we say that $F$ is $(u, \beta)$-dominated whenever there exists $\ell \geqslant 1$ such that

$$
\left\|\partial_{\mathrm{fib}} F^{\ell}(\zeta)\right\| \leqslant \frac{\left(\nu_{u}^{(\ell)}(\pi(\zeta))\right)^{\beta}}{2}, \quad \forall \zeta \in \mathcal{E},
$$

where $\nu_{u}$ is the (multiplicative) cocycle over $f$ given in Definition 2.1.

Analogously, one says that $F$ is said to be $(s, \beta)$-dominated when there exists $\ell \geqslant 1$ such that

$$
\left\|\left(\partial_{\mathrm{fib}} F^{\ell}(\zeta)\right)^{-1}\right\|^{-1} \geqslant 2\left(\nu_{s}^{(\ell)}(\pi(\zeta))\right)^{\beta}, \quad \forall \zeta \in \mathcal{E} .
$$


And $F$ is just said to be $\beta$-dominated if it is simultaneously both $(s, \beta)$ - and $(u, \beta)$-dominated. It is important to remark that the definition of domination implicitly assumes that the dynamics on the base space is given by a hyperbolic homeomorphism $f: M$ ๖.

The following result is a consequence of the classical graph transform arguments used in [HPS77] (see [AV10, Proposition 5.1] for an indication of the proof in this exact context):

Proposition 2.6. If $F$ is an $(s, 1)$-dominated $C^{\mathrm{Lip}, 1}$-fiber bundle over a hyperbolic homeomorphism $f: M$ : then there exists a unique partition of $\mathcal{E}$

$$
\mathcal{W}^{s}=\left\{\mathcal{W}^{s}(\zeta) \subset \mathcal{E}: \zeta \in \mathcal{E}\right\},
$$

which satisfies the following conditions:

(i) for every $\zeta \in \mathcal{E}, \mathcal{W}^{s}(\zeta)$ is the image of a Lipschitz section $W^{s}(\pi(\zeta), f) \rightarrow \mathcal{E}$ whose Lipschitz constant is uniform, i.e. it can be chosen independently of $\zeta$;

(ii) it is F-invariant, i.e.

$$
F\left(\mathcal{W}^{s}(\zeta)\right)=\mathcal{W}^{s}(F(\zeta)), \quad \forall \zeta \in \mathcal{E}
$$

Of course, if $F$ is $(u, 1)$-dominated, a completely analogous result holds and in such a case the "unstable" partition is denoted by $\mathcal{W}^{u}$.

The following result is a combination of Proposition 5.1 and Theorem D of [AV10]:

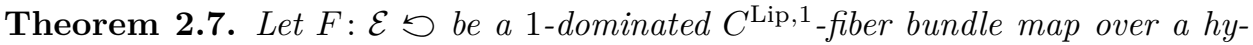
perbolic homeomorphism $f: M \bigcirc$ and let $\hat{\mu}$ be an $F$-invariant probability measure whose projection $\mu:=\pi_{\star}(\hat{\mu})$ to $M$ has local product structure and full support. Then, if $\lambda^{ \pm}(F, \hat{\mu})=0$, the support of $\hat{\mu}$ is saturated by $\mathcal{W}^{s}$ and $\mathcal{W}^{u}$.

2.9. Solving the cohomological equation in Lie groups. We state in this section two results due to Kalinin [Kal11] which will play an important role in our proof of Theorem 3.1. However, it is interesting to remark that our Theorem A is completely independent of those results of Kalinin and, in fact, the results of Livšic [Liv71, Liv72] are enough to deal with the case where the fibers are onedimensional.

The first result of Kalinin is the following one:

Theorem 2.8 (Theorem 1.4 in [Kal11]). Let $A: M \rightarrow \mathrm{GL}_{d}(\mathbb{R})$ be a Hölder cocycle over a transitive hyperbolic homeomorphism $f$. Assume that A satisfies the (POO) condition. Then, the cocycle $A$ has zero Lyapunov exponents with respect to any $f$-invariant ergodic measure on $M$.

Remark 2.9. As it is already observed in [Kal11], the previous result does not make use of the fact that the cocycle acts on a trivial fiber bundle, and so it holds for arbitrary bundle maps. We would like to remark that we can get another proof of Theorem 2.8 as consequence of our Theorem 4.1 below.

In order to show that the solution of cohomological equations are sufficiently regular, we must show that the holonomy maps of certain foliations are smooth. We will show this proving that certain linear cocycles are indeed coboundaries. As it was already mentioned above, that can be done invoking "classical" Livšic theorem when cocyles act on one-dimensional manifolds. In higher dimensions, we need the following Livšic type theorem for linear cocycles due to Kalinin:

Theorem 2.10 (Theorem 1.1 in [Kal11]). Let $A: M \rightarrow \mathrm{GL}_{d}(\mathbb{R})$ a Hölder cocycle over a transitive hyperbolic homeomorphism $f: M$ ๖. Assume that A satisfies 
(POO) condition. Then, for every $x_{0} \in M$, there exists a unique Hölder map $U: M \rightarrow \mathrm{GL}_{d}(\mathbb{R})$ such that

$$
A(x)=U(f(x)) U(x)^{-1}, \quad \forall x \in M,
$$

and $U\left(x_{0}\right)=I d_{\mathbb{R}^{d}}$.

Moreover, there exists a constant $C>0$ depending only on $f$ such that

$$
|U|_{\alpha} \leqslant C|A|_{\alpha}
$$

The following consequence of the previous result will be needed later:

Proposition 2.11. Let $f: M \oslash$ be a hyperbolic homeomorphism and $\left(A_{t}: M \rightarrow\right.$ $\left.\mathrm{GL}_{d}(\mathbb{R})\right)_{t \in N}$ be a continuous family ( $N$ is a topological manifold) of $\alpha$-Hölder cocycles satisfying (POO) condtion. Then, there exists a continuous family of $\alpha$-Hölder transfer functions $\left(U_{t}: M \rightarrow \mathrm{GL}_{d}(\mathbb{R})\right)_{t \in N}$ satisfying

$$
A_{t}(x)=U_{t}(f(x)) U_{t}(x)^{-1}, \quad \forall t \in N, \forall x \in M .
$$

Proof. Consider a point $x_{0} \in M$ whose forward orbit by $f$ is dense and let $U_{t}$ : $M \rightarrow \mathrm{GL}_{d}(\mathbb{R})$ be the unique solution of the cohomological equation $A_{t}(x)=$ $U_{t}(f(x)) U_{t}(x)^{-1}$ with $U_{t}\left(x_{0}\right)=I d$, given by Theorem 2.10. Notice that $\left|U_{t}\right|_{\alpha}$ is uniformly bounded on $t \in N$.

Now we have to show that $U_{t}$ depends continuously on $t \in N$. Let us fix $t_{0} \in N$ and $\varepsilon>0$. Let $n$ be sufficiently large so that the segment of orbit $x_{0}, \ldots, f^{n}\left(x_{0}\right)$ is $\delta$-dense, where

$$
\delta:=\varepsilon\left(6 C \max _{t \in N}\left|A_{t}\right|_{\alpha}\right)^{-\frac{1}{\alpha}},
$$

and $C$ is the positive constant given by Theorem 2.10 .

By continuity of the family $A_{t}$ on $t$, and observing that $U_{t}\left(f^{k}\left(x_{0}\right)\right)=A_{t}^{(k)}\left(x_{0}\right)$ for every $k \geqslant 0$, there exists a neighborhood $V$ of $t_{0}$ such that one has

$$
\left\|U_{t}\left(f^{k}\left(x_{0}\right)\right)-U_{t_{0}}\left(f^{k}\left(x_{0}\right)\right)\right\| \leqslant \frac{\varepsilon}{2}, \quad \text { for } 0 \leqslant k \leqslant n, \forall t \in V .
$$

Invoking the uniform Hölder estimates, we deduce that the $C^{0}$-distance between the functions $U_{t}$ and $U_{t_{0}}$ is smaller than $\varepsilon$, for every $t \in V$.

\section{Domination, Zero Lyapunov EXPONEnts AND COBOUNDARIES}

In this section we study the relation between domination, nullity of Lyapunov exponents and cohomology of bundle maps. The main result we present here is the following

Theorem 3.1. Let $N \rightarrow \mathcal{E} \stackrel{\pi}{\rightarrow} M$ be a $C^{\alpha, 1}$-fiber bundle and $F: \mathcal{E} \circlearrowleft$ be $C^{\alpha, 1}$ bundle map over a $\alpha$-Hölder hyperbolic homeomorphism $f: M$ ๖. Let us assume $F$ satisfies (POO') condition. Then the following statements are equivalent:

(i) $\lambda^{ \pm}(F, \hat{\mu})=0$, for all $\hat{\mu} \in \mathfrak{M}(F)$;

(ii) $F$ is $\alpha$-dominated;

(iii) $F$ is a $C^{\alpha, 1}$-coboundary and, according to $\S 2.6$, the fiber bundle $N \rightarrow \mathcal{E} \stackrel{\pi}{\rightarrow} M$ admits a $C^{\alpha, 1}$-trivialization.

Remark 3.2. We shall use a rather classical trick (see for example [Via08]) which allows us to reduce the general $\alpha$-Hölder case to the Lipschitz one: if $N \rightarrow \mathcal{E} \stackrel{\pi}{\rightarrow}$ $(M, d)$ is a $C^{\alpha, 1}$-fiber bundle, $f:(M, d) \oslash$ is a hyperbolic homeomorphism and $F: \mathcal{E} \bigcirc$ is a $C^{\alpha, 1}$-fiber bundle map which is $\alpha$-dominated, then changing the metric $d$ by $d_{\alpha}$ (see (1)) on $M$, we obtain a $C^{\text {Lip,1 }}$-fiber bundle, $f$ continues to be hyperbolic

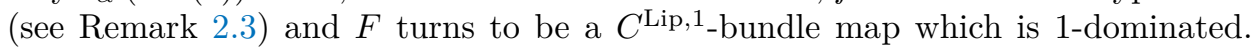

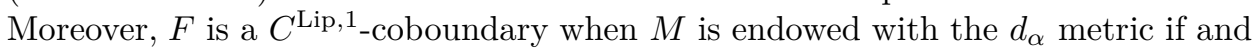
only if it is a $C^{\alpha, 1}$-coboundary when $M$ is equipped with $d$. 
By Remark 3.2, in order to simplify the notation from now on and until the end of this section we shall assume that $\alpha=1=$ Lip.

To start with the proof of Theorem 3.1, we first show that $(i)$ implies $(i i)$. This result maybe belongs to the folklore, but since our context is slightly different from usual ones, we decided to include an outline of the proof:

Proposition 3.3. Let us assume that

$$
\lambda^{ \pm}(F, \hat{\mu})=0, \quad \forall \hat{\mu} \in \mathfrak{M}(F),
$$

\section{Then $F$ is 1-dominated.}

Proof. Let us show that $F$ is $(u, 1)$-dominated. The $(s, 1)$-domination follows from completely analogous arguments. To prove that, we shall only use the hypothesis $\lambda^{+}(F, \hat{\mu})=0$, for every $\hat{\mu} \in \mathfrak{M}(F)$.

Then, let us consider the fiber bundle $\pi_{\mathbb{P}}: \mathbb{P} \rightarrow \mathcal{E}$, where the fiber over an arbitrary $\zeta \in \mathcal{E}$ is given by the projectivized tangent space of the submanifold $\mathcal{E}_{\pi(\zeta)} \subset \mathcal{E}$.

Now, the derivative-along-fiber operator $\partial_{\text {fib }} F$ defined by (5) naturally induces a bundle map $\left[\partial_{\text {fib }} F\right]: \mathbb{P} \circlearrowright$ over $F: \mathcal{E} \supset$.

Then we consider the continuous real cocycle $\psi: \mathbb{P} \rightarrow \mathbb{R}$ over $\left[\partial_{\text {fib }} F\right]$ given by

$$
\psi([v]):=\log \frac{\left\|\partial_{\mathrm{fib}} F \cdot v\right\|_{F(\zeta)}}{\|v\|_{\zeta}}, \quad \forall \zeta \in \mathcal{E}, \forall v \in T \mathcal{E}_{\pi(\zeta)} \backslash\{0\},
$$

where $[v]$ denotes the element of $\mathbb{P}$ induced by $v$.

Now, let $K_{0}, \lambda$ be the constants and $\nu_{u}$ be the multiplicative cocycle associated to $f$ given by Definition 2.1, and suppose $F$ is not $(u, 1)$-dominated. Then, there exists a sequence of points $\left(\zeta_{n}\right)_{n \geqslant 1}$ in $\mathcal{E}$ and a strictly increasing sequence of natural numbers $\left(\ell_{n}\right)_{n \geqslant 1}$ such that

$$
\left\|\partial_{\mathrm{fib}} F^{\ell_{n}}\left(\zeta_{n}\right)\right\| \geqslant \frac{\nu_{u}^{\left(\ell_{n}\right)}\left(\pi\left(\zeta_{n}\right)\right)}{2}, \quad \forall n \geqslant 1
$$

This implies that for each $n \in \mathbb{N}$ we can find $\left[v_{n}\right] \in \mathbb{P}_{\zeta_{n}}$ such that

$$
\psi^{\left(\ell_{n}\right)}\left(\left[v_{n}\right]\right)=\sum_{j=0}^{\ell_{n}-1} \psi\left(\left[\partial_{\mathrm{fib}} F^{j}\right]\left[v_{n}\right]\right)=\log \left\|\partial_{\mathrm{fib}} F^{\ell_{n}}\left(\zeta_{n}\right)\right\| .
$$

Then, by Banach-Alaoglu theorem, there is no lost of generality assuming that there exists $\tilde{\eta} \in \mathfrak{M}(\mathbb{P})$ such that

$$
\frac{1}{\ell_{n}} \sum_{j=0}^{\ell_{n}-1}\left[\partial_{\mathrm{fib}} F^{j}\right]_{\star}\left(\delta_{\left[v_{n}\right]}\right) \rightarrow \tilde{\eta}, \quad \text { as } n \rightarrow \infty,
$$

where the convergence is in the weak- $\star$ topology.

Putting together (8), (9) and (10), we can easily show that

$$
\int_{\mathbb{P}} \psi \mathrm{d} \tilde{\eta}=\lim _{n \rightarrow \infty} \frac{\psi^{\left(\ell_{n}\right)}\left(\left[v_{n}\right]\right)}{\ell_{n}} \geqslant \lim _{n \rightarrow \infty} \frac{1}{\ell_{n}}\left(\log K_{0}+\lambda \ell_{n}-2\right)=\lambda .
$$

Finally, defining $\eta:=\pi_{\mathbb{P} \star}(\tilde{\eta})$, we get $\eta \in \mathfrak{M}(F)$ and from (11) it easily follows

$$
\lambda^{+}(F, \eta) \geqslant \lambda>0 \text {, }
$$

contradicting our hypothesis.

Next we show that $(i i)$ implies $(i i i)$ in Theorem 3.1.

Since we are assuming $F$ is 1-dominated, by Proposition 2.6 we know that we can lift the stable and unstable sets of $f$ to $\mathcal{E}$. We shall need the following properties of them: 
Lemma 3.4. If $\mathcal{W}^{\sigma}$ denotes the lift of $W^{\sigma}$ (with $\sigma \in\{s, u\}$ ), then there exists a constant $K \geqslant 1$ such that

$$
d_{\mathcal{E}}(\zeta, \eta) \leqslant K d(\pi(\zeta), \pi(\eta))
$$

for every $\zeta \in \mathcal{E}, \eta \in \mathcal{W}^{\sigma}(\zeta)$ and such that $\pi(\eta) \in W_{\delta_{0}}^{\sigma}(\pi(\zeta), f)$, where $\delta_{0}$ is the constant associated to $f$ by Definition 2.1 .

Proof. This is a straightforward consequence of Proposition 2.6, i.e. the fact that the elements of $\mathcal{W}^{\sigma}$ are graphs of Lipschitz functions with uniformly bounded constant over the stable and unstable sets of $f$.

Then we need the following result that plays a key role in the construction of solutions for the cohomological equation:

Proposition 3.5. If $F$ is 1-dominated and satisfies (POO') condition, then the closure of every $F$-orbit is the image of a Lipschitz section. More precisely, for every $\zeta \in \mathcal{E}$, there exists a Lipschitz section $V_{\zeta}: \overline{\mathcal{O}_{f}(\pi(\zeta))} \subset M \rightarrow \mathcal{E}$ such that

$$
\overline{\mathcal{O}_{F}(\zeta)}=\left\{V_{\zeta}(y) \in \mathcal{E}: y \in \overline{\mathcal{O}_{f}(\pi(\zeta))}\right\}
$$

Proof. In order to show that the closure of any $F$-orbit coincides with the image of a continuous section of the fiber bundle $N \rightarrow \mathcal{E} \stackrel{\pi}{\rightarrow} M$, it is enough to show the following

Claim 1. For every $\zeta \in \mathcal{E}$ and every $\varepsilon>0$, there exists $\delta>0$ such that

$$
d_{\mathcal{E}}\left(\zeta, F^{n}(\zeta)\right)<\varepsilon
$$

whenever $d\left(\pi(\zeta), f^{n}(\pi(\zeta))\right)<\delta$.

To prove Claim 1, let $\zeta \in \mathcal{E}$ and $\varepsilon>0$ be arbitrary. Then, let us choose $\delta:=\min \left(\delta_{0}, \delta_{1}, \varepsilon(4 c K)^{-1}\right)$, where constants $\delta_{1}$ and $c$ are given by Theorem 2.4 and $K$ is given by Lemma 3.4 .

Then, suppose $n \in \mathbb{N}$ is given such that $d\left(\pi(\zeta), f^{n}(\pi(\zeta))\right)<\delta$. Since $f$ is a hyperbolic homeomorphism and $\delta \leqslant \delta_{1}$, we can apply Theorem 2.4 to guaranty the existence of $p \in \operatorname{Per}(f)$ and $y:=[\pi(\zeta), p] \in M$ satisfying (1), (2) and (3) in Theorem 2.4. Thus, taking into account that the fiber bundle projection $\pi$ is oneto-one on $\mathcal{W}^{u}(\zeta) \subset \mathcal{E}$ and $y \in W^{u}(\pi(\zeta), f)=\pi\left(\mathcal{W}^{u}(\zeta)\right)$, there exists a unique point $\zeta_{y} \in \mathcal{E}_{y} \cap \mathcal{W}^{u}(\zeta)$. Analogously, $\pi$ is one-to-one from $\mathcal{W}^{s}\left(\zeta_{y}\right)$ onto $W^{s}(y)$ and hence, there exists a unique point $\zeta_{p} \in \mathcal{E}_{p} \cap \mathcal{W}^{s}\left(\zeta_{y}\right)$.

Now, observing that $\zeta_{p} \in \mathcal{W}^{s}\left(\zeta_{y}\right)$ and $\zeta_{y} \in \mathcal{W}^{u}(\zeta)$, we can combine Theorem 2.4 and Lemma 3.4 to guaranty that

$$
d_{\mathcal{E}}\left(\zeta, \zeta_{p}\right) \leqslant d_{\mathcal{E}}\left(\zeta, \zeta_{y}\right)+d_{\mathcal{E}}\left(\zeta_{y}, \zeta_{p}\right) \leqslant K[d(\pi(\zeta), y)+d(y, p)]<2 K c \delta \leqslant \frac{\varepsilon}{2}
$$

and

$$
\begin{aligned}
d_{\mathcal{E}}\left(F^{n}(\zeta), F^{n}\left(\zeta_{p}\right)\right) & \leqslant d_{\mathcal{E}}\left(F^{n}(\zeta), F^{n}\left(\zeta_{y}\right)\right)+d_{\mathcal{E}}\left(F^{n}\left(\zeta_{y}\right), F^{n}\left(\zeta_{p}\right)\right) \\
& \leqslant K\left[d\left(f^{n}\left(\pi(\zeta), f^{n}(y)\right)+d\left(f^{n}(y), f^{n}(p)\right)\right]\right. \\
& \leqslant 2 K c d\left(f^{n}(\pi(\zeta)), \pi(\zeta)\right)<2 K c \delta \leqslant \frac{\varepsilon}{2}
\end{aligned}
$$

Finally observe that, since $f^{n}(p)=p$ and $F$ satisfies (POO'), it holds $F^{n}\left(\zeta_{p}\right)=$ $\zeta_{p}$. Then, putting together (12) and (13), we get $d_{\mathcal{E}}\left(\zeta, F^{n}(\zeta)\right)<\varepsilon$, and our claim is proven.

It is interesting to notice that the exponential shadowing given by Anosov closing lemma (Theorem 2.4) was not used in the proof of Proposition 3.5. In fact, the 
classical Shadowing Lemma is enough because the Hölder regularity (in this case Lipschitz) was already used in Lemma 3.4.

Now, let us fix any point $x_{0} \in M$ such that its forward and backward $f$-orbits are dense. Then, by Proposition 3.5, assuming $F$ is 1-dominated, for every $\zeta \in \mathcal{E}_{x_{0}}$ there exists a continuous section $V_{\zeta}: M \rightarrow \mathcal{E}$ such that $\overline{\mathcal{O}_{F}(\zeta)}$ coincides with the image of $V_{\zeta}$. To simplify the notation, the image of the section $V_{\zeta}$ will be denoted by $\mathcal{V}_{\zeta}$, i.e. we define $\mathcal{V}_{\zeta}:=\left\{V_{\zeta}(x) \in \mathcal{E}: x \in M\right\}$, for every $\zeta \in \mathcal{E}_{x_{0}}$.

Then we will show that the family $\left\{\mathcal{V}_{\zeta}\right\}_{\zeta \in \mathcal{E}_{x_{0}}}$ determines a continuous lamination in $\mathcal{E}$. To do this, we first prove the following

Proposition 3.6. Assuming $F$ is 1-dominated, for each $\zeta \in \mathcal{E}_{x_{0}}$ the image of the section $V_{\zeta}$ defined above is saturated by leaves of the lamination $\mathcal{W}^{s}\left(\mathcal{W}^{u}\right.$, respectively.) More precisely, for every $\zeta \in \mathcal{E}_{x_{0}}$ and any $\eta \in \mathcal{V}_{\zeta}$,

$$
\mathcal{W}^{\sigma}(\eta) \subset \mathcal{V}_{\zeta}, \quad \text { for } \sigma \in\{s, u\} \text {. }
$$

Proof. Let us suppose the proposition is not true. Then, there exists some $\zeta \in \mathcal{E}_{x_{0}}$ and $\eta^{\prime} \in \mathcal{V}_{\zeta}$ such that $\mathcal{W}^{s}\left(\eta^{\prime}\right) \notin \mathcal{V}_{\zeta}\left(\mathcal{W}^{u}\left(\eta^{\prime}\right) \notin \mathcal{V}_{\zeta}\right.$, respectively). By continuity of the section $V_{\zeta}$ and the stable (unstable, respec.) lamination, we can choose a point $\eta \in \mathcal{V}_{\zeta}$ such that the forward (backward, respec.) $f$-orbit of $\pi(\eta)$ is dense in $M$ and $\mathcal{W}^{s}(\eta) \notin \mathcal{V}_{\zeta}\left(\mathcal{W}^{u}(\eta) \notin \mathcal{V}_{\zeta}\right.$, respec.) Then, we take a point $\xi \in \mathcal{W}^{s}(\eta) \backslash \mathcal{V}_{\zeta}$ $\left(\xi \in \mathcal{W}^{u}(\eta) \backslash \mathcal{V}_{\zeta}\right.$, respec. $)$. Observe that $\mathcal{O}_{f}^{+}(\pi(\xi))\left(\mathcal{O}_{f}^{-}(\pi(\xi))\right.$, respec.) is dense in $M$. Hence, the section $V_{\xi}$ given by Proposition 3.5 is defined on the whole space $M$. But, since $\xi \in \mathcal{W}^{s}(\eta)\left(\xi \in \mathcal{W}^{u}(\eta)\right.$, respec. $)$, the set $\overline{\mathcal{O}_{F}(\xi)}$ intersects the fiber $\mathcal{E}_{\pi(\xi)}$ at two different points: at $\xi$ and at $V_{\zeta}(\pi(\xi))$, contradicting Proposition 3.5.

Remark 3.7. It is interesting to notice that a less elementary proof of Proposition 3.6 can be easily gotten invoking the topological version of the Invariance Principle of Avila and Viana (see Theorem 2.7). In fact, assuming domination and (POO'), using Theorem 2.8, it can be shown that condition $(i)$ of Theorem 3.1 holds and then the Invariance Principle can be applied.

As a consequence of Proposition 3.6 we get the family $\left\{\mathcal{V}_{\zeta}\right\}_{\zeta \in \mathcal{E}_{x_{0}}}$ is a partition of the total space $\mathcal{E}$, and moreover, a continuous lamination whose leaves are (topologically) transverse to the fibers of the fiber bundle $N \rightarrow \mathcal{E} \stackrel{\pi}{\rightarrow} M$. Thus, we can define the holonomy maps of this lamination as follows: given arbitrary points $x, y \in M$, we define the holonomy map from $x$ to $y$ is defined by

$$
\mathcal{H}_{x, y}: \mathcal{E}_{x} \ni \zeta \mapsto V_{\hat{\zeta}}(y) \in \mathcal{E}_{y},
$$

where $\hat{\zeta}$ is the unique point in $\mathcal{E}_{x_{0}}$ such that $\zeta \in \mathcal{V}_{\hat{\zeta}}$. Observe that, by Proposition 3.6, the holonomy maps are (at least) homeomorphisms. After some additional results, we shall show they are indeed $C^{1}$-diffeomorphisms.

Then we get the following

Proposition 3.8. The fiber bundle map $F: \mathcal{E} \bigcirc$ is a $C^{\mathrm{Lip}, 0}$-coboundary. More precisely, the fiber bundle $N \rightarrow \mathcal{E} \stackrel{\pi}{\rightarrow} M$ admits a continuous trivialization $H: \mathcal{E} \rightarrow$ $M \times N$ that turns the diagram (4) commutative.

Proof. To show that the fiber bundle is trivial, let us consider the map $H: \mathcal{E} \rightarrow$ $M \times N$ given by

$$
H(\zeta):=\left(\pi(\zeta), \operatorname{pr}_{2}\left(\phi_{j}\left(\mathcal{H}_{\pi(\zeta), x_{0}}(\zeta)\right)\right)\right), \quad \forall \zeta \in \mathcal{E},
$$

where $\phi_{j}: U_{j} \rightarrow M \times N$ is any (but fixed) trivializing chart of the fiber bundle $N \rightarrow \mathcal{E} \stackrel{\pi}{\rightarrow} M$ such that $x_{0} \in U_{j}$. Then, since holonomy maps are homeomorphisms, 
it is clear that $H$ itself is a homeomorphism, and since $F\left(\mathcal{V}_{\hat{\zeta}}\right)=\mathcal{V}_{\hat{\zeta}}$, for every $\hat{\zeta} \in \mathcal{E}_{x_{0}}$, we conclude that

as desired.

$$
H(F(\zeta))=\left(f \times i d_{N}\right)(H(\zeta)), \quad \forall \zeta \in \mathcal{E}
$$

Finally, in order to show that $F$ is a $C^{\mathrm{Lip}, 1}$-coboundary, it remains to prove that the map $H: \mathcal{E} \rightarrow M \times N$ constructed in the proof of Proposition 3.8 is indeed a $C^{\text {Lip,1-bundle map. }}$

To do this, it is necessary to show that the holonomy maps defined in (14) are differentiable and this will be gotten invoking Proposition 2.11. To use this result, we first need the following

Lemma 3.9. For every $\zeta \in \mathcal{E}_{x_{0}}$, the section $V_{\zeta}: M \rightarrow \mathcal{E}$ (whose image is $\mathcal{V}_{\zeta}$ ) is Lipschitz.

Proof. It is a straightforward consequence of the fact that the graph of $V_{\zeta}$ is saturated by $\mathcal{W}^{s}$ and $\mathcal{W}^{u}$, which are Lischitz themselves and have local product structure (see (h5) in Definition 2.1).

Now, for every $\zeta \in \mathcal{E}_{x_{0}}$, consider the set

$$
\Xi^{\zeta}:=\bigsqcup_{x \in M} T_{V_{\zeta}(x)} \mathcal{E}_{x}
$$

where $\bigsqcup$ denotes the disjoint-union operator, and the "natural projection" map $\pi^{\zeta}: \Xi^{\zeta} \rightarrow M$ given by $\left(\pi^{\zeta}\right)^{-1}(x)=T_{V_{\zeta}(x)} \mathcal{E}_{x}$, for every $x \in M$. By Lemma 3.9, the set $\Xi^{\zeta}$ can naturally be endowed with an appropriate vector bundle structure

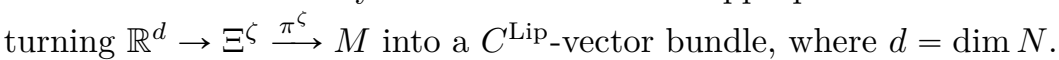

On the other hand, since every leaf $\mathcal{V}_{\zeta}$ is $F$-invariant and $\left.F\right|_{\mathcal{E}_{x}}: \mathcal{E}_{x} \rightarrow \mathcal{E}_{f(x)}$ is a $C^{1}$-diffeomorphism, our fiber bundle map $F$ naturally induces a $C^{\text {Lip }}$-vector bundle map $D F^{\zeta}: \Xi^{\zeta} \bigcirc$ over $f: M \supset$ given by

$$
D F^{\zeta}\left(v_{x}\right)=\partial_{\mathrm{fib}} F\left(V_{\zeta}(x)\right)\left(v_{x}\right), \quad \forall x \in M, \forall v_{x} \in \Xi_{x}^{\zeta}=T_{V_{\zeta}(x)} \mathcal{E}_{x},
$$

where $\partial_{\text {fib }} F$ denotes the (partial) derivative along the fibers defined in $\S 2.7$.

Then we get the following

Proposition 3.10. For every $\zeta \in \mathcal{E}_{x_{0}}$, the vector bundle $\mathbb{R}^{d} \rightarrow \Xi^{\zeta} \stackrel{\pi^{\zeta}}{\longrightarrow} M$ is trivial and the vector bundle map $D F^{\zeta}$ is a $C^{\text {Lip }}$-coboundary, i.e. there exists a $C^{\text {Lip }}$-vector bundle map $U^{\zeta}: \Xi^{\zeta} \rightarrow M \times \mathbb{R}^{d}$ satisfying

$$
U^{\zeta} \circ D F^{\zeta}=\left(f \times I d_{\mathbb{R}^{d}}\right) \circ U^{\zeta} .
$$

Moreover, the family $\left(U^{\zeta}\right)_{\zeta \in \mathcal{E}_{x_{0}}}$ can be chosen to vary continuously on $\zeta$.

Proof. Since $F$ satisfies (POO') condition, $D F^{\zeta}$ must satisfy it, too. Hence, by Theorem 2.8, $D F^{\zeta}$ has zero Lyapunov exponents with respect to any $f$-invariant probability measure. In particular, invoking Proposition 3.8 we conclude that the vector bundle $\mathbb{R}^{d} \rightarrow \Xi^{\zeta} \stackrel{\pi^{\zeta}}{\longrightarrow} M$ is trivial and we can apply Proposition 2.11 to obtain a continuous family $U^{\zeta}$ of solutions, as desired.

Then we get the following

Corollary 3.11. If $F$ is 1-dominated and satisfies (POO'), then there exists $C>0$ such that

$$
\left\|\partial_{\text {fib }} F^{n}(v)\right\| \leqslant C, \quad \forall n \in \mathbb{Z}, \forall \zeta \in \mathcal{E}, \forall v \in T_{\zeta} \mathcal{E}_{\pi(\zeta)} .
$$

Proof. This is a straightforward consequence of Propositions 3.10 and 2.11.

Then we finally get 
Proposition 3.12. The holonomy maps given by (14) are differentiable and con-

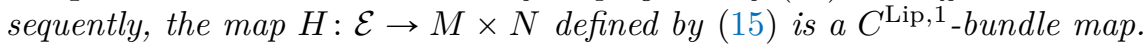

Proof. Given arbitrary points $x, y \in M$, we need to show that the holonomy map $\mathcal{H}_{x, y}: \mathcal{E}_{x} \rightarrow \mathcal{E}_{y}$ associated to the lamination $\{\mathcal{V}\}_{\zeta \in \mathcal{E}_{x_{0}}}$, which is clearly a homeomorphism, is indeed a $C^{1}$-diffeomorphism.

To do this, first observe that since each leaf of the lamination $\left\{\mathcal{V}_{\zeta}\right\}_{\zeta \in \mathcal{E}_{x_{0}}}$ is $F$ invariant, it holds

$$
\mathcal{H}_{f^{m}(x), f^{n}(x)}=\left.F^{n-m}\right|_{\mathcal{E}_{f^{m}(x)}}, \quad \forall x \in \mathcal{E}, \forall m, n \in \mathbb{Z} .
$$

Consequently, holonomy maps between any two points of the same $f$-orbit are indeed $C^{1}$-diffeomorphisms.

To deal with the general case, let $x, y \in M$ be arbitrary points and consider two trivializing charts $\varphi_{i}: \pi^{-1}\left(U_{i}\right) \rightarrow U_{i} \times N$, with $i=1$, 2, such that $x \in U_{1}$ and $y \in U_{2}$. Recalling we have chosen $x_{0} \in M$ so that its forward $f$-orbit is dense in $M$, we can find two sequences of natural numbers $\left(m_{i}\right)$ and $\left(n_{i}\right)$ such that $U_{1} \ni f^{m_{i}}\left(x_{0}\right) \rightarrow x$ and $U_{2} \ni f^{n_{i}}\left(x_{0}\right) \rightarrow y$, as $i \rightarrow \infty$.

Then, for each $i \geqslant 1$, let us define $\mathcal{H}_{i} \in \operatorname{Diff}^{1}(N)$ by

$$
\mathcal{H}_{i}(p):=\operatorname{pr}_{2} \circ \varphi_{2} \circ \mathcal{H}_{f^{m_{i}}\left(x_{0}\right), f^{n_{i}}\left(x_{0}\right)} \circ \varphi_{1}^{-1}\left(f^{m_{i}}\left(x_{0}\right), p\right),
$$

and $\mathcal{H} \in \operatorname{Homeo}(N)$ by

$$
\mathcal{H}(p)=\operatorname{pr}_{2} \circ \varphi_{2} \circ \mathcal{H}_{x, y} \circ \varphi_{1}^{-1}(x, p),
$$

for every $p \in N$. We want to show $\mathcal{H} \in \operatorname{Diff}^{1}(N)$, too.

By continuity of the lamination $\left\{\mathcal{V}_{\zeta}\right\}_{\zeta \in \mathcal{E}_{x_{0}}}$, when $i \rightarrow \infty, \mathcal{H}_{i} \rightarrow \mathcal{H}$ pointwisely. By Corollary 3.11 and Arzelà-Ascoli theorem, we conclude $\mathcal{H}_{i} \rightarrow \mathcal{H} C^{0}$-uniformly. On the other hand, by Propositions 3.10 and 2.11, we have the sequence of derivatives $\left(D \mathcal{H}_{i}(p)\right)_{i \geqslant 1}$ is also convergent, for each $p \in N$. Consequently, $\mathcal{H}$ is $C^{1}$ and then, $\mathcal{H}_{x, y}: \mathcal{E}_{x} \rightarrow \mathcal{E}_{y}$ is a diffeomorphism, as desired.

Finally, it remains to show that (iii) implies $(i)$ in Theorem 3.1. But this is

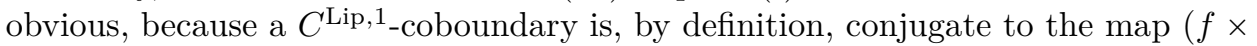

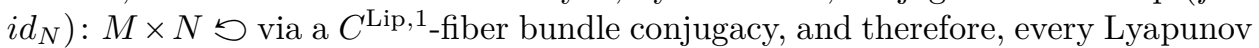
exponent must vanish.

\section{Domination as a Consequence of POO COndition}

In this section we shall review some contexts where condition (POO) alone implies that the cocycle is dominated, and as a consequence of Theorem 3.1, it is a coboundary.

We start proving Theorem B which follows from Theorem 3.1 and Katok closing lemma [Kat80]:

Proof of Theorem B. Let $S \rightarrow \mathcal{E}=M \times S \stackrel{\pi}{\rightarrow} M$ denote the trivial fiber bundle and $F: M \times S \sqsubseteq$ be the $C^{1+\alpha}$ skew-product over $f$ induced by $\Phi$ as in (3). Oberserve that $F$ satisfies (POO').

Let us suppose there exists an $F$-invariant ergodic probability measure $\hat{\mu}$ such that $\lambda^{+}(F, \hat{\mu}) \neq 0$.

Since $\Phi$ takes values in the group of area-preserving diffeomorphisms of $S$, by Oseledets theorem we know that

$$
\lambda^{-}(F, \hat{\mu})+\lambda^{+}(F, \hat{\mu})=0 .
$$

So, we have

$$
\lambda^{-}(F, \hat{\mu})<0<\lambda^{+}(F, \hat{\mu}),
$$


and since $f: M ๖$ is an Anosov diffeomorphism, this implies $\hat{\mu}$ is a hyperbolic measure for $F$ (i.e. all its Lyapunov exponents given by Oseledets theorem are different from zero).

So, applying Katok closing lemma [Kat80, Corollary 4.3], we conclude that $F$ exhibits a hyperbolic periodic point. But, by $\left(\mathrm{POO}^{\prime}\right)$, if $\zeta_{0} \in \mathcal{E}$ is periodic with $F^{n}\left(\zeta_{0}\right)=\zeta_{0}$, then $F^{n}(\zeta)=\zeta$, for every $\zeta \in \mathcal{E}_{\pi \zeta_{0}}$, and so $\zeta_{0}$ is not hyperbolic, getting a contradiction.

The amount of regularity required in the fiber direction is essential in our argument and it is the usual one in Pesin's theory which allows to obtain a subexponential neighborhoods of a regular orbit with good estimates on the bundles of the Oseledet's splitting (see [KH96, Suplement]). The recent examples of [BCS13] show that improving this regularity requires new ideas which should not be different from the general case of $\operatorname{Diff}^{1}(N)$ cocycles with arbitrary $N$.

In order to prove Theorem A, we need the following result that should be considered the main one of this section:

Theorem 4.1. Let $N \rightarrow \mathcal{E} \stackrel{\pi}{\rightarrow} M$ be a $C^{\alpha, 1}$-fiber bundle, $F: \mathcal{E} \bigcirc$ be a $C^{\alpha, 1}$ bundle map over an $\alpha$-Hölder hyperbolic homeomorphism $f: M$ ○. If there exists an ergodic measure $\hat{\mu} \in \mathfrak{M}(F)$ with $\lambda^{+}(F, \hat{\mu})<0$, then there exists $\zeta_{0} \in \operatorname{Per}(F)$ which is uniformly contracting along the fiber, i.e. if $n>0$ denotes the period of $\zeta_{0}$, then all the eigenvalues of the linear map $\partial_{\mathrm{fib}} F^{n}\left(\zeta_{0}\right): T_{\zeta_{0}} \mathcal{E}_{\pi\left(\zeta_{0}\right)} \bigcirc$ have modulus strictly smaller than 1 .

It is interesting to remark that applying Theorem 4.1 to the natural action induced by a linear cocycle on a suitable Grasmannian fiber bundle (corresponding to the dimension of the subspace with largest Lyapunov exponent), one can reprove Kalinin's result on approximation ${ }^{3}$ of Lyapunov exponents [Kal11, Theorem 1.4].

Now, we can prove Theorem A as a combination of Theorems 3.1 and 4.1:

Proof of Theorem $A$. Let $\mathbb{R} / \mathbb{Z} \rightarrow \mathcal{E}=M \times \mathbb{R} / \mathbb{Z} \stackrel{\pi}{\rightarrow} M$ denote the trivial fiber bundle and $F: M \times \mathbb{R} / \mathbb{Z} \bigcirc$ be the skew-product over $f$ induced by $\Phi$ as in (3). Since $\Phi$ satisfies $(\mathrm{POO}), F$ satisfies (POO').

Hence, for every $\zeta \in \operatorname{Per}(F)$ with $F^{n}(\zeta)=\zeta$ it clearly holds $\partial_{\text {fib }} F^{n}=D \Phi^{(n)} \equiv i d$ and consequently, all the eigenvalues are equal to 1 . So, applying Theorem 4.1 to $F$ and $F^{-1}$ we get

$$
-\lambda^{+}\left(F^{-1}, \hat{\mu}\right)=\lambda^{-}(F, \mu) \leqslant 0 \leqslant \lambda^{+}(F, \hat{\mu}) .
$$

But since the fibers are one-dimensional, we can apply Birkhoff ergodic theorem to conclude that $\lambda^{-}(F, \hat{\mu})=\lambda^{+}(F, \hat{\mu})$. Consequently, $\lambda^{-}(F, \hat{\mu})=\lambda^{+}(F, \hat{\mu})=0$ and by Theorem 3.1, $F$ is a $C^{\alpha, 1}$-coboundary, as desired.

4.1. Proof of Theorem 4.1. From the uniform continuity of $\partial_{\mathrm{fib}} F$ and $f$, it easily follows

Lemma 4.2. For every $\delta>0$, there exists $\chi>0$ such that for every $\eta, \xi \in \mathcal{E}$ satisfying

then one has

$$
d_{\mathcal{E}}\left(F^{i}(\eta), F^{i}(\xi)\right) \leqslant \chi, \quad \text { for every } i \in\{0, \ldots k\}
$$

$$
\prod_{i=0}^{k-1}\left\|\partial_{\mathrm{fib}} F\left(F^{i}(\eta)\right)\right\| \leqslant e^{k \delta} \prod_{i=0}^{k-1}\left\|\partial_{\mathrm{fib}} F\left(F^{i}(\xi)\right)\right\| .
$$

\footnotetext{
${ }^{3}$ The statement above only allows to show that if $\Phi: M \rightarrow \mathrm{GL}_{d}(\mathbb{R})$ is an $\alpha$-Hölder cocycle satisfying the (POO) then every measure has zero Lyapunov exponents, however, the results below allow to recover the complete result if desired.
} 
Along the proof we shall assume that $\lambda^{+}:=\lambda^{+}(F, \hat{\mu})<0$.

It is a classical fact that one can choose measurable adapted metrics which see the contraction at each iterate (see for example Proposition 8.2 of [ABC11]):

Lemma 4.3. For every $\varepsilon>0$ there exists an integer $N>0$ and a measurable function $A: \mathcal{E} \rightarrow[1,+\infty)$ such that:

- The sequence $\left(A\left(F^{n}(\zeta)\right)_{n \in \mathbb{Z}}\right.$ varies sub-exponentially (i.e. one has that for $\hat{\mu}$-almost every $\zeta \in \mathcal{E}$ the sequence $\frac{1}{|n|} \log \left|A\left(F^{n}(\zeta)\right)\right|$ converges to 0 as $|n| \rightarrow$ $\infty)$.

- If we denote $\|\cdot\|_{\zeta}^{\prime}$ to the metric in $T_{\zeta} \mathcal{E}_{\pi(\zeta)}$ defined as:

$$
\|v\|_{\zeta}^{\prime}=\sum_{0 \leqslant k \leqslant N} e^{-k\left(\lambda^{+}+\varepsilon\right)} A\left(F^{k}(\zeta)\right)\left\|\partial_{\mathrm{fib}} F^{k}(\zeta) \cdot v\right\|_{F^{k}(\zeta)}
$$

then, for $\hat{\mu}$ almost every $\zeta \in \mathcal{E}$ and every $v \in T_{\zeta} \mathcal{E}_{\pi(\zeta)}$ one has that

$$
\left\|\partial_{\mathrm{fib}} F(\zeta) \cdot v\right\|_{F(\zeta)}^{\prime} \leqslant e^{\left(\lambda^{+}+\varepsilon\right)}\|v\|_{\zeta}^{\prime}
$$

We shall fix $\varepsilon<\min \left\{-\frac{\lambda^{+}}{5}, \frac{\alpha \lambda}{2}\right\}$, where $\alpha$ is the Hölder exponent of $F$ and $\lambda$ is the hyperbolicity constant appearing in Theorem 2.4. Consider the function $A$ and the metric $\|\cdot\|^{\prime}$ given by the previous lemma and let us fix them from now on.

Using this metric, it is also standard to show that one can define sub-exponential neighborhoods (sometimes called Pesin charts) of typical points with respect to $\hat{\mu}$ such that the dynamics in those neighborhoods behaves similarly to the derivative (see for example [KH96, Supplement]).

For $\zeta \in \mathcal{E}$ we shall consider the exponential map exp $: T_{\zeta} \mathcal{E}_{\pi(\zeta)} \rightarrow \mathcal{E}_{\pi(\zeta)}$ where the distances in $T_{\zeta} \mathcal{E}_{\pi(\zeta)}$ are measured with respect to the metric $\|\cdot\|_{\zeta}^{\prime}$. We denote by $B_{\zeta}^{\prime}(r)$ the ball of radius $r$ centered at 0 in $T_{\zeta} \mathcal{E}_{\pi(\zeta)}$.

Lemma 4.4. There exists a measurable function $\rho: \mathcal{E} \rightarrow(0,+\infty)$ such that if $\varphi_{\zeta}=\left.\exp \right|_{B_{\zeta}^{\prime}(\rho(\zeta))}$ then we have that for $\hat{\mu}$-almost every point one has that $\varphi_{F(\zeta)}^{-1} \circ$ $F \circ \varphi_{\zeta}: B_{\zeta}^{\prime}(\rho(\zeta)) \rightarrow B_{F(\zeta)}^{\prime}(\rho(F(\zeta)))$ contracts vectors by a factor of at least $e^{\left(\lambda^{+}+2 \varepsilon\right)}$. Moreover, it holds that the sequence $\rho\left(F^{n}(\zeta)\right)$ is sub-exponential and can be chosen so that $e^{-\varepsilon} \rho(\zeta)<\rho(F(\zeta))<e^{\varepsilon} \rho(\zeta)$.

It is relevant to remark here the fact that the sub-exponential growth of the function $\rho$ is essential in Pesin's theory and it is where the Hölder regularity of the derivative is usually used. Here, since we are working with measures whose Lyapunov exponents are all negative, $C^{1}$-regularity in the fibers is enough.

Remark 4.5. Notice that there is a measurable function $D$ which, associates to each $\zeta \in \mathcal{E}$ an isometry $D_{\zeta}:\left(T_{\zeta} \mathcal{E}_{\pi(\zeta)},\|\cdot\|_{\zeta}\right) \rightarrow\left(T_{\zeta} \mathcal{E}_{\pi(\zeta)},\|\cdot\|_{\zeta}^{\prime}\right)$. When this linear map is seen as a transformation from $\left(T_{\zeta} \mathcal{E}_{\pi(\zeta)},\|\cdot\|_{\zeta}\right)$ to itself, the norm and co-norm of $D_{\zeta}$ are bounded by a number depending only on $A(\zeta)$.

Using Luisin's Theorem on approximation of measurable functions by continuous ones (see for example [KH96, Supplement]) one obtains a compact set $X \subset \mathcal{E}$ of positive $\hat{\mu}$-measure such that functions $A$ and $\rho$ are continuous on $X$ and, thus, bounded (we define $A_{X}:=\sup _{\zeta \in X} A(\zeta)$ and $\rho_{X}:=\inf _{\zeta \in X} \rho(x)$ ).

Consider a point $\zeta_{0} \in X$ which is recurrent inside $X$, i.e. there exists $n_{j} \rightarrow \infty$ such that $F^{n_{j}}\left(\zeta_{0}\right) \rightarrow \zeta_{0}$ and $F^{n_{j}}\left(\zeta_{0}\right) \in X$ for every $j>0$.

Let us write $x_{0}:=\pi\left(\zeta_{0}\right)$ and, for each $j>0$, let $p_{j} \in \operatorname{Fix}\left(f^{n_{j}}\right)$ be the periodic point of $f$ given by Anosov Closing Lemma (Theorem 2.4). One has that:

$$
d\left(f^{i}\left(x_{0}\right), f^{i}\left(p_{j}\right)\right) \leqslant c e^{-\lambda \min \left\{i, n_{j}-i\right\}} d\left(f^{n_{j}}\left(x_{0}\right), x_{0}\right), \quad \text { for } i=0, \ldots, n_{j} .
$$


where $c, \lambda>0$ are the constants given in Theorem 2.4. Notice that $d\left(f^{n_{j}}\left(x_{0}\right), x_{0}\right) \leqslant$ $d_{\mathcal{E}}\left(F^{n_{j}}\left(\zeta_{0}\right), \zeta_{0}\right) \rightarrow 0$.

Fix $\delta<\varepsilon$ and let $\chi$ be the constant given by Lemma 4.2 for such a $\delta$.

The main step in the proof is the following

Lemma 4.6. For $n_{j}$ large enough, there exists a small open ball $B_{j} \subset \mathcal{E}_{p_{j}}$ such that

$$
F^{n_{j}}\left(\overline{B_{j}}\right) \subset B_{j} .
$$

Moreover, $\operatorname{diam}\left(F^{i}\left(B_{j}\right)\right) \leqslant \chi / 2$ and $d_{\mathcal{E}}\left(F^{i}\left(B_{j}\right), F^{i}\left(\zeta_{0}\right)\right) \leqslant \chi / 2$, for every $0 \leqslant i \leqslant$ $n_{j}$.

Let us now conclude the proof of Theorem 4.1 assuming this lemma:

By (18), we know there exists $\xi_{j} \in B_{j}$ such that $F^{n_{j}}\left(\xi_{j}\right)=\xi_{j}$, and it also holds $d_{\mathcal{E}}\left(F^{i}\left(\xi_{j}\right), F^{i}\left(\zeta_{0}\right)\right) \leqslant \chi$, for all $0 \leqslant i \leqslant n_{j}$. Hence, applying Lemma 4.2 and the fact that $\zeta_{0}, F^{n_{j}}\left(\zeta_{0}\right) \in X$, we prove that $\xi_{j}$ is uniformly contracting along the fiber, as desired.

So, it only remains to prove Lemma 4.6:

Proof of Lemma 4.6. Since there exists a trivializing chart containing $x_{0}$ and $p_{j}$, there exists a point $\zeta_{j} \in \mathcal{E}_{p_{j}}$ such that $d_{\mathcal{E}}\left(\zeta_{j}, \zeta_{0}\right)=d\left(p_{j}, x\right)$.

From the choice of $x_{0}$ and $p_{j}$, if $n_{j}$ is large enough, we can always assume that both $f^{i}\left(x_{0}\right)$ and $f^{i}\left(p_{j}\right)$ lie in the same trivializing chart for $0 \leqslant i \leqslant n_{j}$. So, fixing a trivializing chart containing $f^{i}\left(x_{0}\right)$ and $f^{i}\left(p_{j}\right)$, we have a projection $\operatorname{pr}_{2}: \mathcal{E}_{f^{i}\left(p_{j}\right)} \rightarrow \mathcal{E}_{f^{i}\left(x_{0}\right)}$. Given two points $\xi \in \mathcal{E}_{f^{i}\left(x_{0}\right)}$ and $\eta \in \mathcal{E}_{f^{i}\left(p_{j}\right)}$ such that $\xi, \operatorname{pr}_{2}(\eta) \in B_{F^{i}\left(\zeta_{0}\right)}^{\prime}\left(\rho\left(F^{i}\left(\zeta_{0}\right)\right)\right)$, we can define $d_{\mathcal{E}}^{\prime}(\xi, \eta):=d(\pi(\xi), \pi(\eta))+d^{\prime}\left(\xi, \operatorname{pr}_{2}(\eta)\right)$, were $d^{\prime}$ is the distance in $B_{F^{i}\left(\zeta_{0}\right)}^{\prime}\left(\rho\left(F^{i}\left(\zeta_{0}\right)\right)\right)$ induced by the norm $\|\cdot\|_{F^{i}\left(\zeta_{0}\right)}^{\prime}$.

For $1 \leqslant k \leqslant n_{j}$, and assuming that $d_{\mathcal{E}}^{\prime}\left(F^{k-1}\left(\zeta_{j}\right), F^{k-1}\left(\zeta_{0}\right)\right) \leqslant \min \left\{\rho\left(F^{k-1}\left(\zeta_{0}\right)\right), \frac{\chi}{2}\right\}$, we can invoke Lemma 4.2 to get

$$
\begin{aligned}
& d_{\mathcal{E}}^{\prime}\left(F^{k}\left(\zeta_{j}\right), F^{k}\left(\zeta_{0}\right)\right)=d_{\mathcal{E}}^{\prime}\left(F\left(F^{k-1}\left(\zeta_{j}\right)\right), F\left(F^{k-1}\left(\zeta_{0}\right)\right)\right) \leqslant \\
& \leqslant \hat{c} e^{\varepsilon \min \left\{k, n_{j}-k\right\}} d_{C^{1}}\left(F_{f^{k-1}\left(p_{j}\right)}, F_{f^{k-1}\left(x_{0}\right)}\right)+ \\
& +e^{\delta}\left\|\partial_{\mathrm{fib}} F\left(F^{k-1}\left(\zeta_{0}\right)\right)\right\|^{\prime} d_{\mathcal{E}}^{\prime}\left(F^{k-1}\left(\zeta_{j}\right), F^{k-1}\left(\zeta_{0}\right)\right),
\end{aligned}
$$

where the constant $\hat{c}$ only depends on $A_{X}$ and $\rho_{X}$. The factor $\hat{c} e^{\varepsilon \min \left\{k, n_{j}-k\right\}}$ appears to take into account the distortion in the new metric, which is bounded by $\hat{c}$ at the points $\zeta_{0}, F^{n_{j}}\left(\zeta_{0}\right) \in X$ and the change of the distortion at each iterate is bounded by $e^{\varepsilon}$.

Now, let us define the sequences $a_{k}:=\hat{c} e^{\varepsilon \min \left\{k, n_{j}-k\right\}} d_{C^{1}}\left(F_{f^{k-1}\left(p_{j}\right)}, F_{f^{k-1}\left(x_{0}\right)}\right)$ and $b_{k}:=e^{\delta}\left\|\partial_{\mathrm{fib}} F\left(F^{k-1}\left(\zeta_{0}\right)\right)\right\|^{\prime}$. Observe that $b_{k} \leqslant e^{\left(\lambda^{+}+3 \varepsilon\right)}<1$, for every $k \geqslant 1$.

By induction and applying estimate (19), one gets

$$
d_{\mathcal{E}}^{\prime}\left(F^{k}\left(\zeta_{j}\right), F^{k}\left(\zeta_{0}\right)\right) \leqslant \sum_{i=1}^{k} a_{i}\left(\prod_{j=i+1}^{k} b_{j}\right) \leqslant \sum_{i=1}^{k} a_{i} e^{(k-i)\left(\lambda^{+}+3 \varepsilon\right)}, \quad \forall k \geqslant 1 .
$$

One can estimate the size of $a_{i}$ as follows (here is where Hölder continuity of $F$ is essential):

$$
a_{i} \leqslant e^{\varepsilon \min \left\{i, n_{j}-i\right\}} c^{\prime} e^{-\alpha \lambda \min \left\{i, n_{j}-i\right\}} d\left(x_{0}, f^{n_{j}}\left(x_{0}\right)\right)^{\alpha}, \quad \text { for } 0 \leqslant i \leqslant n_{j},
$$

where $c^{\prime}>0$ depends on the constant $c$ appearing in Anosov closing lemma (Theorem 2.4), the Hölder norm of the $C^{\alpha, 1}$-bundle map $F$ and the constant $\hat{c}$ which was defined above. 
Choosing $n_{j}$ so that $d\left(x_{0}, f^{n_{j}}\left(x_{0}\right)\right)$ is sufficiently small and recalling that $\varepsilon<$ $\frac{1}{2} \alpha \lambda$, thus we can perform the induction and to ensure that the iterates $F^{k}\left(\zeta_{j}\right)$ of the point $\zeta_{j}$ remain always close enough to $F^{k}\left(\zeta_{0}\right)$.

Using the estimate of Lemma 4.2, one conclude that there is a ball $B_{j}$ with the desired properties.

\section{Some QUESTIONS}

In this section we pose some questions that emerge rather naturally from our results and remain open. The firs one, taking into account Theorem 3.1, should be the only remaining step necessary to prove Livšic theorem for cocycles of diffeomorphisms in any dimension:

Question 5.1. Let $N \rightarrow \mathcal{E} \stackrel{\pi}{\rightarrow} M$ be a $C^{\alpha, 1}$-fiber bundle and $F: \mathcal{E} \bigcirc$ be $C^{\alpha, 1}$ bundle map over a hyperbolic $C^{\alpha}$-homeomorphism $f: M \bigcirc$. Suppose $F$ satisfies (POO'). Then, is it true that $F$ is $\alpha$-dominated?

The second one concerns the validity of Livšic type results for more general groups:

Question 5.2. Does there exists a complete metric group $G$ such that Livšic theorem does not hold for $G$-cocyles? More precisely: do there exist a hyperbolic homeomorphism $f: M \bigcirc$ and $a C^{\alpha}$-cocycle $\Phi: M \rightarrow G$ such that $\Phi$ satisfies (POO) but it is not a $G$-coboundary?

\section{REFERENCES}

[ABC11] F. Abdenur, C. Bonatti, and S. Crovisier, Nonuniform hyperbolicity for $C^{1}$-generic diffeomorphisms, Israel J. Math. 183 (2011), 1-60. MR 2811152

[AV10] A. Avila and M. Viana, Extremal Lyapunov exponents: an invariance principle and applications, Invent. Math. 181 (2010), no. 1, 115-189. MR 2651382

[BCS13] C. Bonatti, S. Crovisier, and K. Shinohara, The $C^{1+\alpha}$ hypothesis in Pesin Theory revisited, J. Mod. Dyn. 7 (2013), no. 4, 605-618. MR 3177774

[dlLW10] R. de la Llave and A. Windsor, Livšic theorems for non-commutative groups including diffeomorphism groups and results on the existence of conformal structures for Anosov systems, Ergodic Theory Dynam. Systems 30 (2010), no. 4, 1055-1100. MR 2669410

[dILW11] Smooth dependence on parameters of solutions to cohomology equations over Anosov systems with applications to cohomology equations on diffeomorphism groups, Discrete Contin. Dyn. Syst. 29 (2011), no. 3, 1141-1154. MR 2773168

[GG14] G. Grabarnik and M. Guysinsky, Livšic theorem for Banach rings, available at arXiv:1408.5639v1, 2014.

[HPS77] M. W. Hirsch, C. C. Pugh, and M. Shub, Invariant manifolds, Lecture Notes in Mathematics, Vol. 583, Springer-Verlag, Berlin, 1977. MR 0501173 (58 \#18595)

[Jou88] J.-L. Journé, A regularity lemma for functions of several variables, Rev. Mat. Iberoamericana 4 (1988), no. 2, 187-193. MR 1028737 (91j:58123)

[Kal11] B. Kalinin, Livšic theorem for matrix cocycles, Ann. of Math. 173 (2011), no. 2, 10251042. MR 2776369 (2012b:37082)

[Kat80] A. Katok, Lyapunov exponents, entropy and periodic orbits for diffeomorphisms, Inst. Hautes Études Sci. Publ. Math. 51 (1980), 137-173. MR 573822 (81i:28022)

[KH96] A. Katok and B. Hasselblatt, Introduction to the modern theory of dynamical systems, vol. 54, Cambridge Univ. Pr., 1996.

[KK96] A. Katok and A. Kononenko, Cocycle stability for partially hyperbolic systems, Math. Res. Letters 3 (1996), 191-210.

[KN11] A. Katok and V. Niţică, Rigidity in higher rank abelian group actions. Volume I, Cambridge Tracts in Mathematics, vol. 185, Cambridge University Press, Cambridge, 2011, Introduction and cocycle problem. MR 2798364 (2012i:37039)

[Koc13] A. Kocsard, On the cohomological $C^{0}$-(in)stability, Bulletin of the Brazilian Mathematical Society 44 (2013), no. 3, 489-495.

[Liv71] A. N. Livšic, Certain properties of the homology of $Y$-systems, Mat. Zametki 10 (1971), 555-564. MR 0293669 
[Liv72] _ Cohomology of dynamical systems, Izv. Akad. Nauk SSSR Ser. Mat. 36 (1972), $1296-1320$

[Mañ87] R. Mañé, Ergodic theory and differentiable dynamics, Ergebnisse der Mathematik und ihrer Grenzgebiete (3), vol. 8, Springer-Verlag, Berlin, 1987, Translated from the Portuguese by Silvio Levy. MR 889254 (88c:58040)

[NP13] A. Navas and M. Ponce, A Livsic type theorem for germs of analytic diffeomorphisms, Nonlinearity 26 (2013), no. 1, 297-305.

[NT95] V. Niţică and A. Török, Cohomology of dynamical systems and rigidity of partially hyperbolic actions of higher-rank lattices, Duke Math. J. 79 (1995), no. 3, 751-810. MR 1355183 (96k:58168)

[NT96] _ Regularity results for the solutions of the Livsic cohomology equation with values in diffeomorphism groups, Ergodic Theory Dynam. Systems 16 (1996), no. 2, 325-333. MR 1389627

[Via08] M. Viana, Almost all cocycles over any hyperbolic system have nonvanishing Lyapunov exponents, Ann. of Math. 167 (2008), no. 2, 643-680. MR 2415384 (2009i:37080)

[Wil13] A. Wilkinson, The cohomological equation for partially hyperbolic diffeomorphisms, Astérisque (2013), no. 358, 75-165. MR 3203217

Instituto de Matemática e Estatística - Universidade Federal Fluminense. Rua Mário Santos Braga S/N, 24.020-140 Niterói, RJ, Brasil

$U R L$ : www.professores.uff.br/kocsard

E-mail address: akocsard@id.uff.br

CMAT, Facultad de Ciencias, Universidad de la República, Uruguay

$U R L$ : www. cmat.edu.uy/ rpotrie

E-mail address: rpotrie@cmat.edu.uy 